\title{
Alcohol effects on synaptic transmission in periaqueductal gray dopamine neurons
}

\author{
Chia Li ${ }^{a}, b$, Nora M. McCall ${ }^{b}$, Alberto J. Lopez ${ }^{b}$, and Thomas L. Kash ${ }^{b, c,{ }^{*}}$ \\ ${ }^{a}$ Curriculum in Neurobiology, University of North Carolina-Chapel Hill School of Medicine, Chapel \\ Hill, NC 27599, USA \\ bBowles Center for Alcohol Studies, University of North Carolina-Chapel Hill, Chapel Hill, NC \\ 27599, USA \\ 'Department of Pharmacology, University of North Carolina-Chapel Hill, Chapel Hill, NC 27599, \\ USA
}

\begin{abstract}
The role of dopamine (DA) signaling in regulating the rewarding properties of drugs, including alcohol, has been widely studied. The majority of these studies, however, have focused on the DA neurons located in the ventral tegmental area (VTA), and their projections to the nucleus accumbens. DA neurons within the ventral periaqueductal gray (vPAG) have been shown to regulate reward but little is known about the functional properties of these neurons, or how they are modified by drugs of abuse. This lack of knowledge is likely due to the highly heterogeneous cell composition of the vPAG, with both $\gamma$-amino-butyric acid (GABA) and glutamate neurons present in addition to DA neurons. In this study, we performed whole-cell recordings in a THeGFP transgenic mouse line to evaluate the properties of vPAG-DA neurons. Following this initial characterization, we examined how both acute and chronic alcohol exposure modify synaptic transmission onto vPAG-DA neurons. We found minimal effects of acute alcohol exposure on GABA transmission, but a robust enhancement of glutamatergic synaptic transmission in vPAGDA. Consistent with this effect on excitatory transmission, we also found that alcohol caused an increase in firing rate. These data were in contrast to the effects of chronic intermittent alcohol exposure, which had no significant impact on either inhibitory or excitatory synaptic transmission on the vPAG-DA neurons. These data add to a growing body of literature that points to alcohol having both region-dependent and cell-type dependent effects on function.
\end{abstract}

\section{Keywords}

Periaqueductal gray; Dopamine; Alcohol; GABA; Glutamate; Emotional disorder

\section{Introduction}

Alcohol-use disorders are an enormous public health problem, and understanding the neurochemical systems involved in regulating the actions of alcohol can provide insight for the development of more effective treatments. Numerous reports have suggested that the subjective responses to acute alcohol exposure are related to the risk of development of alcohol-use disorders (Morean \& Corbin, 2010). As such, understanding the actions of acute

(C) 2013 Elsevier Inc. All rights reserved.

*Corresponding author. Bowles Center for Alcohol Studies, University of North Carolina-Chapel Hill, 104 Manning Drive, Chapel Hill, NC 27599, USA. Tel.: +1 919966 7116. tkash@email.unc.edu (T.L. Kash). 
alcohol exposure is an important area of investigation. Animal studies have shown that acute alcohol exposure can induce locomotor stimulation (Humeniuk, White, \& Ong, 1993; Koob, 1992; Kornetsky, Bain, Unterwald, \& Lewis, 1988) and is also anxiolytic (Morales-Mulia et al., 2012; Sharko, Kaigler, Fadel, \& Wilson, 2013). Alcohol exposure also modifies pain perception and the anti-nociceptive effects of opiates (Peris, Peiro, Hernandez, \& de la Parte, 2005). Chronic alcohol exposure produces very different behavioral effects during withdrawal. Locomotor activity has been reported to decrease (Broadwater, Varlinskaya, \& Spear, 2011), and anxiety-like behaviors are observed (Kliethermes, 2005) during the withdrawal period. Other withdrawal-induced behaviors include aggression (Echevarria, Toms, \& Jouandot, 2011; Heinz, Beck, Meyer-Lindenberg, Sterzer, \& Heinz, 2011), anhedonia (Fukushiro et al., 2012), insomnia (Brower \& Perron, 2010), and increased sensitivity to pain (Gatch, 2009; Jochum, Boettger, Burkhardt, Juckel, \& Bar, 2010). Given the diversity of these behavioral outcomes, there are likely multiple brain regions and their projection regions that are modified by alcohol exposure.

A large body of evidence suggests that dopamine (DA) signaling plays a critical role in mediating the rewarding aspects of acute alcohol exposure. Much of this research has focused on the mesolimbic DA system, which is composed of DA neurons in the ventral tegmental area (VTA) that project to the nucleus accumbens (Gonzales, Job, \& Doyon, 2004; Wise, 2006). However, recent studies have shown that dopamine signaling in other brain regions critical to alcohol abuse, such as the extended amygdala (Eiler, Seyoum, Foster, Mailey, \& June, 2003), can play an important role in alcohol reward. Interestingly, the extended amygdala receives a strong dopaminergic projection from the A10dc DA neurons, located in the ventral periaqueductal gray (vPAG), as well as dorsal raphe nucleus (DRN) (Hasue \& Shammah-Lagnado, 2002; Meloni, Gerety, Knoll, Cohen, \& Carlezon, 2006). The vPAG is particularly relevant as a target for the acute actions of alcohol as it has been implicated in the regulation of arousal (Heinz et al., 2011; Jia et al., 2012), anxiety (Devall \& Lovick, 2010; Mendes-Gomes \& Nunes-de-Souza, 2009; Spiacci, Coimbra, \& Zangrossi, 2012), sleep (Lu, Jhou, \& Saper, 2006), pain (Flores, El Banoua, GalanRodriguez, \& Fernandez-Espejo, 2004; Freund et al., 2011; Mendes-Gomes, Amaral, \& Nunes-de-Souza, 2011; Yang et al., 2011), and opiate reward (Flores, Galan-Rodriguez, Ramiro-Fuentes, \& Fernandez-Espejo, 2006). Despite the potential behavioral relevance of vPAG-DA neurons to the actions of alcohol, there have been no studies examining either the properties of these neurons, or the ability of alcohol to modify their function. In this study, we utilized a TH-eGFP transgenic mouse to selectively record from DA neurons in the vPAG and evaluated the impact of both acute and chronic alcohol exposure on synaptic transmission.

With the knowledge that vPAG-DA neurons project to the extended amygdala and that pharmacological blockade of dopamine receptors in these regions can modulate alcoholdrinking behavior, we investigated the effects of both acute and chronic alcohol exposure on synaptic function in vPAG-DA neurons. Briefly, we found that alcohol did not modify miniinhibitory postsynaptic currents (mIPSCs) in vPAG-DA neurons, but increased miniexcitatory post-synaptic current (mEPSC) frequency. Consistent with these synaptic effects, we found that acute alcohol exposure increased firing of vPAG-DA neurons. Interestingly, chronic alcohol exposure did not alter synaptic function in these neurons.

\section{Materials and methods}

\section{Animals and husbandry}

Adult male TH-eGFP mice on a Swiss Webster background (aged between 5 and 9 weeks) were bred and used in accordance with an animal use protocol approved by the University of North Carolina - Chapel Hill (IACUC). Mice were group-housed in our colony room under 
a 12:12-h light cycle, with lights on at 7:00 AM daily. Mice were given ad libitum access to rodent chow and water. Mating pairs of mice were created by GENSAT and obtained from the Mutant Mouse Regional Resource Center in North Carolina. In the TH-eGFP mouse line, the genome was modified to contain multiple copies of a modified bacterial artificial chromosome in which an eGFP reporter gene was inserted immediately upstream of the coding sequence of the gene for tyrosine hydroxylase (TH). Data presented here were obtained from the transgenic mice maintained in-house.

\section{Electrophysiology brain slice preparation}

Mice were decapitated under isoflurane anesthesia and their brains were rapidly removed and placed in ice-cold sucrose artificial cerebrospinal fluid (ACSF) (in mM): 194 sucrose, $20 \mathrm{NaCl}, 4.4 \mathrm{KCl}, 2 \mathrm{CaCl}_{2}, 1 \mathrm{MgCl}_{2}, 1.2 \mathrm{NaH}_{2} \mathrm{PO}_{4}, 10.0$ glucose, and $26.0 \mathrm{NaHCO}_{3}$ saturated with $95 \% \mathrm{O}_{2} / 5 \% \mathrm{CO}_{2}$. Three hundred micron slices were prepared using a Leica VT1200 vibratome (Wetzlar, Germany).

\section{Slice whole-cell electrophysiology}

Brain slices containing the PAG were obtained and stored at approximately $30^{\circ} \mathrm{C}$ in a heated, oxygenated holding chamber containing artificial cerebrospinal fluid (ACSF) (in $\mathrm{mmol} / \mathrm{L}$ ): $124 \mathrm{NaCl}, 4.4 \mathrm{KCl}, 2 \mathrm{CaCl}_{2}, 1.2 \mathrm{MgSO}_{4}, 1 \mathrm{NaH}_{2} \mathrm{PO}_{4}, 10.0$ glucose, and 26.0 $\mathrm{NaHCO}_{3}$ before being transferred to a submerged recording chamber maintained at approximately $30^{\circ} \mathrm{C}$ (Warner Instruments, Hamden, Connecticut). Recording electrodes (3$5 \mathrm{M} \Omega$ ) were pulled with a Flaming-Brown Micropipette Puller (Sutter Instruments, Novato, CA) using thin-walled borosilicate glass capillaries. During inhibitory transmission experiments, recording electrodes were filled with (in mmol/L) $70 \mathrm{KCl}, 65 \mathrm{~K}^{+}$-gluconate, 5 $\mathrm{NaCl}, 10$ 4-(2-hydroxyethyl)-1-piperazineethanesulfonic acid, 2 QX-314, 0.6 EGTA, 4 ATP, 0.4 GTP, $\mathrm{pH}$ 7.4, 290-295 mOsmol. During excitatory transmission experiments, recording electrodes were filled with (in mmol/L) 117 o-gluconic acid, $118 \mathrm{CsOH}, 20$ HEPES, 0.4 EGTA, 5 TEA, $2 \mathrm{MgCl}_{2}, 4 \mathrm{Na}_{2} \mathrm{APT}, 0.4 \mathrm{Na}_{2} \mathrm{GPT}, 2 \mathrm{QX}-314$. To measure $\mathrm{I}_{\mathrm{h}}$ current, cells were held at $-140 \mathrm{mV}$ for a 1-s duration immediately after breaking into the membrane and analyzed for the final $100 \mathrm{~ms}$ of this voltage step. In voltage-clamp experiments, cells were held at $-70 \mathrm{mV}$ and inhibitory post-synaptic currents (IPSCs) were pharmacologically isolated with $3 \mathrm{mmol} / \mathrm{L}$ kynurenic acid, to block a-amino-3-hydroxy-5-methyl-4-isoxazolepropionic acid (AMPA) and $N$-methyl-o-aspartate (NMDA) receptor-dependent postsynaptic current. Excitatory post-synaptic currents (EPSCs) were pharmacologically isolated by adding $25 \mu \mathrm{mol} / \mathrm{L}$ picrotoxin to block $\gamma$-amino-butyric acid (GABA) receptor-dependent current. To isolate miniature inhibitory post-synaptic currents (mIPSCs), tetrodotoxin (0.5 $\mu \mathrm{mol} / \mathrm{L}$ ) was added to the perfusing ACSF solutions described above. During electrophysiology experiments with chronic intermittent alcohol exposure, mini-inhibitory (cells held at $+10 \mathrm{mV}$ ) and excitatory (cells held at $-55 \mathrm{mV}$ ) transmissions were examined within the same cells using the following internal solution (in $\mathrm{mmol} / \mathrm{L}$ ): $135 \mathrm{Cs}-$ methanesulfonate, $10 \mathrm{KCl}, 10 \mathrm{HEPES}, 1 \mathrm{MgCl}_{2}$, 0.2 EGTA, $4 \mathrm{MgATP}, 0.3 \mathrm{Na}_{2} \mathrm{GPT}, 20$ phosphocreatine. In firing-rate experiments, we used cell-attached experiments where the cell membrane was not ruptured, with the seal maintained under $50 \mathrm{M} \Omega$, while recording electrodes were filled with (in mmol/L): $135 \mathrm{~K}^{+}$-gluconate, $5 \mathrm{NaCl}, 2 \mathrm{MgCl}_{2}, 10 \mathrm{HEPES}$, 0.6 EGTA, $4 \mathrm{Na}_{2} \mathrm{APT}, 0.4 \mathrm{Na}_{2} \mathrm{GPT}$. Signals were acquired via a Multiclamp 700B amplifier (Molecular Devices, Sunnyvale, California), digitized at $20 \mathrm{kHz}$, filtered at $3 \mathrm{kHz}$, and analyzed using Clampfit 10.2 software (Molecular Devices). Input resistance and access resistance were continuously monitored during experiments. Experiments in which changes in access resistance were greater than $20 \%$ were not included in the data analysis. 


\section{Chronic intermittent alcohol vapor paradigm}

Adult mice were exposed to alcohol vapor or air in La Jolla Alcohol Research chambers for $16 \mathrm{~h}$ per day ( $16 \mathrm{~h}$ on, from 4:00 PM to 8:00 AM the following day; $8 \mathrm{~h}$ off) for 2 cycles of 4 days in and 3 days off. Alcohol vapor was created by bubbling $95 \%$ alcohol in a vaporizer at a rate of 4-6 L/min air; the vapor was administered at a flow rate of approximately $15 \mathrm{~L} /$ min air to each individually sealed cage. Alcohol levels were adjusted to reach animal blood alcohol concentration (BAC) above $200 \mathrm{mg} / \mathrm{dL}$, as well as monitored using a Breathalyzer ${ }^{\circledR}$ at the beginning and end of every exposure. To achieve intoxication BAC levels, alcoholexposed mice were injected with $10 \mathrm{~mL} / \mathrm{kg}$ of $68.1 \mathrm{mg} / \mathrm{kg}$ of the alcohol dehydrogenase inhibitor pyrazole combined with $1.5 \mathrm{~g} / \mathrm{kg} 20 \%(\mathrm{v} / \mathrm{v})$ alcohol in saline; air-exposed mice received $10 \mathrm{~mL} / \mathrm{kg}$ of $68.1 \mathrm{mg} / \mathrm{kg}$ pyrazole in saline. Electrophysiology was performed $48 \mathrm{~h}$ after the mice exited the chamber from the last cycle.

\section{Immunohistochemistry}

Mice were anesthetized with Avertin and perfused transcardially with chilled $0.01 \mathrm{M}$ phosphate-buffered saline (PBS), immediately followed by $4 \%$ paraformaldehyde phosphate-buffered saline. Brains were extracted and post-fixed in $4 \%$ paraformaldehyde for $24 \mathrm{~h}$ and immersed in a $30 \%$ sucrose solution for $48 \mathrm{~h}$, both at $4{ }^{\circ} \mathrm{C}$. Coronal sections $45 \mu \mathrm{m}$ in thickness were collected using a Leica VT1000S vibratome (Leica Microsystems, Nussloch, Germany) and stored in a 50\% glycerol solution at $-20{ }^{\circ} \mathrm{C}$ until immunohistochemistry was performed. Slices were rinsed for $5 \mathrm{~min}$ in chilled PBS, followed by a 10-min incubation in $0.1 \%$ Triton X-100 in PBS solution, two 5-min PBS washes, and a 30-min incubation in $0.5 \%$ Triton X-100 in PBS solution. After a 1-h incubation in a blocking solution made of $0.1 \%$ Triton X-100/10\% Normal Donkey Serum in PBS, the tissue was then incubated for $48 \mathrm{~h}$ at $4{ }^{\circ} \mathrm{C}$ with their respective primary antibodies diluted in $0.1 \%$ Triton X-100/10\% Normal Donkey Serum in PBS blocking solution (anti-Tyrosine Hydroxylase [1:500], Pel-Freez P60101-0, Lot 28632; anti-Green Fluorescent Protein [1:500], Aves Laboratories, GFP-1020). Slices were rinsed three times for $10 \mathrm{~min}$ in chilled PBS before incubating for $24 \mathrm{~h}$ at $4{ }^{\circ} \mathrm{C}$ in their respective secondary antibodies diluted in PBS (Alexa Fluor 647 Donkey anti-Sheep [1:800], TH; Alexa Fluor 488 Donkey anti-Chicken [1:200], GFP; Jackson Immuno Research). Tissue was rinsed four times for $10 \mathrm{~min}$ in chilled PBS, and mounted using Vecta-Shield Mounting Medium (Vector Laboratories, Burlingame, CA, H-1000) prior to image collection.

\section{Statistics}

Effects of drugs during electrophysiological recordings were evaluated by comparing the magnitude of the dependent measure (mIPSC or mEPSC frequency and amplitude) between the baseline and wash-on (when drug had reached maximal effect at $10 \mathrm{~min}$ ) periods using paired $t$ tests. The effects of antagonists/blockers on the ability of drugs to modulate synaptic transmission were compared using $t$ tests during the washout period. All values given for drug effects throughout the article are presented as mean \pm SEM.

\section{Results}

\section{Basal electrophysiology properties: vPAG vs. VTA}

We first wanted to confirm that the TH-eGFP mouse line would report correctly for DA neurons in the vPAG. To examine this, we performed dual-label immunofluorescence and looked for overlap of TH and eGFP in the vPAG of the reporter (Fig. 1). We found that 69.6 $\pm 4.5 \%$ of eGFP-positive neurons were co-localized with TH $(n=2)$ animals. Having determined the fidelity of this TH-eGFP reporter line for DA neurons in the vPAG, we then examined the membrane capacitance and resistance in eGFP-positive neurons in the vPAG. 
We found that DA neurons in the $\mathrm{vPAG}(n=35)$ have an average membrane resistance of $866.7 \pm 72.5 \mathrm{M} \Omega$, and a membrane capacitance of $16.6 \pm 1.0 \mathrm{pF}$. In addition, the DA neurons in the vPAG had an average $\mathrm{I}_{\mathrm{h}}$ current of $-8.1 \pm 2.4 \mathrm{pA}$, with most neurons (21 out of 35 ) in the vPAG lacking any measurable hyperpolarization current.

\section{Acute alcohol modification of GABAergic transmission in the VPAG}

We next evaluated the effects of $50 \mathrm{mM}$ alcohol on GABAergic synaptic transmission. We selected a concentration based on previous studies that showed effective modification of inhibitory currents in the VTA (Theile, Morikawa, Gonzales, \& Morrisett, 2008, 2011). We found no effects $(n=6)$ on miniature IPSC (mIPSC) frequency $(100.4 \pm 27.6 \%$ of baseline, Fig. 2A and B), amplitude ( $85.4 \pm 17.0 \%$ of baseline, Fig. 2 C), or decay $(98.4 \pm 4.7 \%$ of baseline, Fig. 2D). Further, acute alcohol exposure had no effects on evoked IPSC transmission (data not shown). Our data suggest that synaptic GABAergic transmission in vPAG-DA neurons is not modified by alcohol. However, previous studies have shown that alcohol can potently modify extra-synaptic $\mathrm{GABA}_{\mathrm{A}}$ receptors (Jia, Chandra, Homanics, \& Harrison, 2008). Thus, we examined the impact of $50 \mathrm{mM}$ alcohol on holding current and the standard deviation of the noise, two measures that are related to tonic $\mathrm{GABA}_{\mathrm{A}}$ receptormediated currents (Jia et al., 2008). We found that $50 \mathrm{mM}$ alcohol does not alter the holding current or cause a shift in the noise (Fig. 2E), suggesting that neither synaptic nor extrasynaptic GABAergic transmission are modified by acute alcohol exposure in the vPAG. As recent studies have found that the actions of alcohol on dopamine neurons can depend on intact network function (Guan et al., 2012), we next assessed the ability of alcohol to modify spontaneous GABAergic transmission (Fig. 3A and B). In these experiments glutamatergic transmission was blocked, but tetrodotoxin was excluded from the bath solution to allow for action-potential dependent network activity. We found that a 10-min bath application of 50 $\mathrm{mM}$ alcohol $(n=10)$ significantly $(p<0.05)$ decreased the amplitude $(88.7 \pm 15.5 \%$ of baseline, Fig. 3D) of spontaneous inhibitory post-synaptic current (sIPSC), but had no effect on frequency $(87.3 \pm 42.2 \%$, Fig. $3 \mathrm{C})$.

\section{Acute alcohol exposure modification of miniature excitatory post-synaptic current in the VPAG}

Having established that acute alcohol exposure had minimal effects on inhibitory inputs to vPAG-DA neurons, we next examined the impact of acute alcohol exposure on excitatory glutamatergic synaptic function (Fig. 4A and B). We found that a 10-min bath application of $50 \mathrm{mM}$ alcohol $(n=7)$ significantly $(p=0.02)$ increased mEPSC frequency $(144.9 \pm 17.7 \%$ of baseline, Fig. 4C), but had no effects on amplitude (93.3 $\pm 4.6 \%$ of baseline, Fig. 4D). Interestingly, this effect did not appear to reverse over the course of the 10-min washout. Taken together, these results demonstrate that acute alcohol exposure has no effects on inhibitory inputs, but facilitates excitatory inputs onto vPAG dopamine neurons via a presynaptic mechanism.

\section{Acute alcohol modification of vPAG dopamine neuron cell firing rate}

After finding minimal effects of acute alcohol exposure on inhibitory synaptic inputs, but finding an increase in excitatory synaptic inputs, we investigated the effects of alcohol on overall firing rate using the loose cell-attached recording configuration. In these experiments, we did not block any synaptic transmission, and thus assessed the impact of alcohol on the circuits that regulate vPAG-DA neuron firing. We found that the average firing rate of the last $5 \mathrm{~min}$ of the $10-\mathrm{min} 50 \mathrm{mM}$ alcohol $(2.5 \pm 0.6 \mathrm{~Hz}, n=7$, Fig. $5 \mathrm{~B}$ and C) bath application is significantly ( $p=0.04)$ higher $(32.6 \pm 10.1 \%$ increase) when compared to baseline $(2.0 \pm 0.5 \mathrm{~Hz}$, Fig. 5A). This effect was not reversible with a 15-min washout, similar to our data in mEPSCs. In addition, alcohol application had no significant 
effects ( $n=7$, Fig. 5D) on firing rate in the presence of the excitatory AMPA receptor antagonist NBQX $(10 \mu \mathrm{M})$. These results suggest that acute alcohol exposure increases the firing of vPAG-DA neurons via an enhancement of excitatory inputs.

\section{Chronic intermittent alcohol vapor modification of synaptic transmission in the VPAG}

Having found that acute alcohol exposure could enhance glutamatergic drive onto vPAGDA neurons, we next investigated the effects of chronic intermittent vapor alcohol exposure on both the mini-inhibitory and mini-excitatory inputs onto vPAG-DA neurons. It has been previously shown that 2 cycles of chronic intermittent alcohol vapor exposure has both behavioral and electrophysiological effects. Specifically, mice undergoing this exposure paradigm exhibit enhanced alcohol-seeking behavior (Becker \& Lopez, 2004; Griffin, Lopez, Yanke, Middaugh, \& Becker, 2009), as well as altered plasticity in the BNST (Silberman, Matthews, \& Winder, 2013; Wills et al., 2012), a target of vPAG-DA neurons. We recorded both mEPSC (Fig. 6A) and mIPSC (Fig. 6B) transmission within the same cell and compared the frequency, amplitude, and excitatory/inhibitory (E/I) ratio between airexposed animals $(n=8)$ and alcohol-exposed animals $(n=7)$. Surprisingly, our results showed no differences in basal frequency (Fig. 6C), amplitude (Fig. 6D), and E/I ratio (Fig. $6 \mathrm{E})$ between control and alcohol groups. In addition, we examined basal cell properties (data not shown) of both groups and found no difference in membrane capacitance, resistance, and holding potential. Taken together, these results suggest that two weeks of chronic alcohol vapor exposure does not alter the basal synaptic properties of vPAG-DA neurons at this time point.

\section{Discussion}

Understanding how alcohol modifies neurochemically defined neurons in distinct brain regions is crucial to understanding alcohol's effects on behavior. This study focused on determining the actions of alcohol on a population of DA neurons in the vPAG that have been implicated in a wide variety of behaviors. We identified dopamine neurons using the TH-eGFP transgenic mouse line and found approximately $70 \%$ of GFP-positive cells were co-labeled with TH. While there is a concern for over-reporting, it is also a possibility that some GFP-positive cells express TH at levels that are too low to be observed through the immunofluorescent techniques. Further, it is critical to note that a recent paper from the Ungless group showed similar results (Dougalis et al., 2012). Having confirmed the fidelity of this reporter, we then characterized vPAG-DA neuron membrane properties.

Interestingly, we observed little to no hyperpolarization-activated currents, similar to what was observed in a recent study (Dougalis et al., 2012). Historically, researchers have used the presence of $\mathrm{I}_{\mathrm{h}}$ current to identify dopamine neurons in the VTA (Ford, Mark, \& Williams, 2006; Klink, de Kerchoved'Exaerde, Zoli, \& Changeux, 2001; Korotkova, Ponomarenko, Brown, \& Haas, 2004; Lammel et al., 2008). While some studies were unable to establish a consistent relationship between dopaminergic neurons and $\mathrm{I}_{\mathrm{h}}$ currents (Margolis, Lock, Hjelmstad, \& Fields, 2006), other studies have suggested that the magnitude of the $\mathrm{I}_{\mathrm{h}}$ current varies with target projection areas, as well as sub-regions of DA neuron-populated sites (Ford et al., 2006; Lammel et al., 2008). Recent studies have found dopamine neurons located in the medial portion of the VTA that display small $\mathrm{I}_{\mathrm{h}}$ currents (Hnasko, Hjelmstad, Fields, \& Edwards, 2012), similar to our results in vPAG-DA neurons. Given the physiological and anatomical similarities between these populations of neurons, it is possible that they play similar roles in alcohol- and stress-related behaviors. Indeed, preliminary results from our group suggest that similar to the neurons in the medial VTA, the TH neurons in the vPAG also express vGlut2. Future studies using optogenetic approaches will more clearly examine this interesting possibility. 
Having characterized the basic properties of vPAG-DA neurons, we next evaluated the effects of alcohol on synaptic transmission. We found that concentrations of bath-applied alcohol that had previously been shown to increase GABA release in the VTA, had no effect on mIPSCs on vPAG-DA neurons. Our result contrasts with previous studies from the VTA that have demonstrated alcohol can enhance both spontaneous and miniature GABA transmission (Guan et al., 2012; Theile et al., 2008). The ability of alcohol to enhance GABA transmission in the VTA, however, appears to depend on the sub-region of the VTA, with anterior-VTA DA neurons showing an alcohol-induced increase in GABA transmission and posterior-VTA DA neurons showing a reduction in spontaneous but not miniature GABA transmission (Guan et al., 2012). Thus, our results demonstrating a lack of alcohol effects on mIPSCs in the vPAG are similar to posterior-VTA DA neurons. Following this, we investigated how alcohol might impact extra-synaptic receptors. Curiously, we found that $50 \mathrm{mM}$ alcohol did not alter the holding current of these neurons, suggesting a lack of effect on extra-synaptic currents. While we did not see evidence for a modification here, this could be due to the exquisite sensitivity of extra-synaptic receptors for alcohol. However, in a series of pilot experiments, we examined the ability of THIP, a compound that can selectively activate extra-synaptic receptors, to modulate holding current in vPAG-DA neurons and found no effect, suggesting that these neurons do not express these receptors. Because of the lack of effect on mini IPSCs and holding current, we investigated the effect of alcohol on spontaneous IPSCs, as it was shown in the posterior VTA that alcohol significantly reduced sIPSCs. We found similar results as Guan et al. that alcohol caused a significant but minimal decrease in spontaneous inhibitory transmission; however, the decrease in amplitude we observed appears less robust than the effect in the posterior VTA. This could be due to some differences in effects of alcohol on the surrounding network and inputs. Taken together, these results suggest that vPAG-DA neurons could be similarly modified by alcohol as the posterior-VTA DA neurons, and may have a partially overlapping set of GABA inputs, either from local interneurons or distal projections such as the BNST and the central amygdala.

We next examined the effects of alcohol on excitatory glutamatergic inputs in vPAG dopamine neurons. We found that bath application of alcohol increased mEPSC frequency, with no effect on amplitude, suggesting an increase in glutamate release. Numerous studies have shown that acute alcohol exposure decreases glutamatergic transmission in many brain regions implicated in alcohol abuse, such as the central nucleus of the amygdala (CeA) (Roberto et al., 2004). The ability of acute alcohol exposure to enhance glutamatergic transmission in the vPAG was strikingly similar to that found in the VTA, where dopamine signaling mediates the enhancement of glutamatergic input by alcohol (Deng, Li, Zhou, \& Ye, 2008). Our observation of alcohol having minimal inhibitory effects on GABAergic inputs but an enhancement in glutamatergic transmission led us to investigate alcohol's effects on overall firing rate. We found an increase in firing rate upon application of acute alcohol, with this alcohol effect diminished in the presence of NBQX, suggesting this increase in firing rate is AMPA receptor dependent. Taken together with our previous data, we speculate that via these alterations in function, acute alcohol exposure may lead to increased dopamine release in the terminal regions, such as the BNST. This is interesting, as DA signaling in the BNST can modulate alcohol-drinking behavior (Eiler et al., 2003; Kash, 2012).

To further investigate the role alcohol plays in modifying vPAG-DA neurons and their target regions, we examined the synaptic effects of chronic intermittent alcohol vapor exposure for 2 cycles. This paradigm has been shown to enhance the development of long-term potentiation in the BNST, via modification of the excitatory NMDA receptors extrasynaptically (Wills et al., 2012), as well as modification of CRF-dependent increase of sEPSCs in the BNST (Silberman et al., 2013). Given the knowledge that chronic alcohol 
exposure modifies synapses and functions in the BNST, one of the extended amygdala structures that interact with the vPAG and impact behaviors critical to alcohol addiction, our findings were surprising. We found no effects of chronic intermittent alcohol exposure on either the inhibitory or excitatory inputs in the vPAG-DA neurons. Perhaps a more profound systemic insult than the 2 cycles of intermittent alcohol exposure presented is required to affect the synaptic functions and to produce a detectable network effect. While considering the possibility that the 2-cycle vapor alcohol exposure paradigm could impact synaptic alterations differentially in a strain-dependent manner, it is interesting to note that during alcohol exposure, the Swiss Webster mice used in these experiments displayed a robust behavioral alteration toward aggression. Alternatively, it is possible that there are alterations in transmitter release in downstream regions such as the BNST that would engage CRF signaling leading to the alterations in function (Silberman et al., 2013). While our study focused on changes in synaptic transmission, it is also possible that chronic alcohol exposure is altering expression of neuromodulatory receptors in the BNST. Chronic alcohol exposure has been shown to alter opioid peptides in the vPAG (Lindholm, Ploj, Franck, \& Nylander, 2000), as well as in other brain regions that interact with the vPAG (Chang et al., 2010). This is interesting, as opioids have been shown to influence alcohol-related behaviors (Margolis, Fields, Hjelmstad, \& Mitchell, 2008; Walker \& Koob, 2008; Walker, Zorrilla, \& Koob, 2011). Thus, although a direct network effect was not observed, chronic intermittent alcohol exposure could have an impact on modulatory peptides in the vPAG and how they regulate synaptic transmission.

It is currently unclear how alcohol-induced modifications of vPAG-DA neurons alter behavior. Given the prominent role that the vPAG plays in sleep and pain processing, it is tempting to speculate that actions of alcohol on this circuit are involved in these processes. Future studies utilizing optogenetics to probe pathway-defined plasticity, as well as applying designer receptors exclusively activated by designer drugs (DREADD) to characterize pathway and cell-type specific modulation will likely shed light on this exciting possibility.

\section{Conclusions}

Here, we characterized the cell membrane properties as well as effects of alcohol exposure on vPAG-DA neurons. Acute alcohol exposure enhanced glutamate inputs and had inhibitory effects on GABA inputs, resulting in a net increase in firing rate. Chronic intermittent alcohol vapor exposure had no effects on GABA nor glutamate transmission onto the vPAG-DA neurons. Taken together, these results add to the growing body of literature pointing toward discrete effects of alcohol on defined cell types in the brain.

\section{References}

Becker HC, Lopez MF. Increased ethanol drinking after repeated chronic ethanol exposure and withdrawal experience in C57BL/6 mice. Alcoholism: Clinical and Experimental Research. 2004; 28:1829-1838.

Broadwater M, Varlinskaya EI, Spear LP. Chronic intermittent ethanol exposure in early adolescent and adult male rats: effects on tolerance, social behavior, and ethanol intake. Alcoholism: Clinical and Experimental Research. 2011; 35:1392-1403.

Brower KJ, Perron BE. Prevalence and correlates of withdrawal-related insomnia among adults with alcohol dependence: results from a national survey. American Journal on Addictions. 2010; 19:238244. [PubMed: 20525030]

Chang GQ, Barson JR, Karatayev O, Chang SY, Chen YW, Leibowitz SF. Effect of chronic ethanol on enkephalin in the hypothalamus and extra-hypothalamic areas. Alcoholism: Clinical and Experimental Research. 2010; 34:761-770. 
Deng C, Li KY, Zhou C, Ye JH. Ethanol enhances glutamate transmission by retrograde dopamine signaling in a postsynaptic neuron/synaptic bouton preparation from the ventral tegmental area. Neuropsychopharmacology. 2008; 34:1233-1244. [PubMed: 18784647]

Devall AJ, Lovick TA. Differential activation of the periaqueductal gray by mild anxiogenic stress at different stages of the estrous cycle in female rats. Neuropsychopharmacology. 2010; 35:11741185. [PubMed: 20072120]

Dougalis AG, Matthews GA, Bishop MW, Brischoux F, Kobayashi K, Ungless MA. Functional properties of dopamine neurons and coexpression of vasoactive intestinal polypeptide in the dorsal raphe nucleus and ventro-lateral periaqueductal grey. European Journal of Neuroscience. 2012; 36:3322-3332. [PubMed: 22925150]

Echevarria DJ, Toms CN, Jouandot DJ. Alcohol-induced behavior change in zebrafish models. Reviews in Neuroscience. 2011; 22:85-93.

Eiler WJ 2nd, Seyoum R, Foster KL, Mailey C, June HL. D1 dopamine receptor regulates alcoholmotivated behaviors in the bed nucleus of the stria terminalis in alcohol-preferring $(\mathrm{P})$ rats. Synapse. 2003; 48:45-56. [PubMed: 12557272]

Flores JA, El Banoua F, Galan-Rodriguez B, Fernandez-Espejo E. Opiate anti-nociception is attenuated following lesion of large dopamine neurons of the periaqueductal grey: critical role for D1 (not D2) dopamine receptors. Pain. 2004; 110:205-214. [PubMed: 15275769]

Flores JA, Galan-Rodriguez B, Ramiro-Fuentes S, Fernandez-Espejo E. Role for dopamine neurons of the rostral linear nucleus and periaqueductal gray in the rewarding and sensitizing properties of heroin. Neuropsychopharmacology. 2006; 31:1475-1488. [PubMed: 16292327]

Ford CP, Mark GP, Williams JT. Properties and opioid inhibition of mesolimbic dopamine neurons vary according to target location. Journal of Neuroscience. 2006; 26:2788-2797. [PubMed: $16525058]$

Freund W, Wunderlich AP, Stuber G, Mayer F, Steffen P, Mentzel M, et al. The role of periaqueductal gray and cingulate cortex during suppression of pain in complex regional pain syndrome. Clinical Journal of Pain. 2011; 27:796-804. [PubMed: 21593662]

Fukushiro DF, Saito LP, Mari-Kawamoto E, Aramini TC, Costa JM, Josino FS, et al. Withdrawal from repeated treatment with ethanol induces a protracted decrease in novelty-seeking behavior and enhancement of environmental habituation in mice. Pharmacology Biochemistry \& Behavior. 2012; 101:132-137.

Gatch MB. Ethanol withdrawal and hyperalgesia. Current Drug Abuse. 2009; 2:41-50.

Gonzales RA, Job MO, Doyon WM. The role of mesolimbic dopamine in the development and maintenance of ethanol reinforcement. Pharmacology \& Therapeutics. 2004; 103:121-146. [PubMed: 15369680]

Griffin WC 3rd, Lopez MF, Yanke AB, Middaugh LD, Becker HC. Repeated cycles of chronic intermittent ethanol exposure in mice increases voluntary ethanol drinking and ethanol concentrations in the nucleus accumbens. Psychopharmacology (Berlin). 2009; 201:569-580. [PubMed: 18791704]

Guan Y, Xiao C, Krnjevic K, Xie G, Zuo W, Ye JH. GABAergic actions mediate opposite ethanol effects on dopaminergic neurons in the anterior and posterior ventral tegmental area. Journal of Pharmacology and Experimental Therapeutics. 2012; 341:33-42. [PubMed: 22209891]

Hasue RH, Shammah-Lagnado SJ. Origin of the dopaminergic innervation of the central extended amygdala and accumbens shell: a combined retrograde tracing and immunohistochemical study in the rat. Journal of Comparative Neurology. 2002; 454:15-33. [PubMed: 12410615]

Heinz AJ, Beck A, Meyer-Lindenberg A, Sterzer P, Heinz A. Cognitive and neurobiological mechanisms of alcohol-related aggression. Nature Reviews Neuroscience. 2011; 12:400-413.

Hnasko TS, Hjelmstad GO, Fields HL, Edwards RH. Ventral tegmental area glutamate neurons: electrophysiological properties and projections. Journal of Neuroscience. 2012; 32:15076-15085. [PubMed: 23100428]

Humeniuk RE, White JM, Ong J. The role of GABAB receptors in mediating the stimulatory effects of ethanol in mice. Psychopharmacology (Berlin). 1993; 111:219-224. [PubMed: 7870956] 
Jia F, Chandra D, Homanics GE, Harrison NL. Ethanol modulates synaptic and extrasynaptic GABAA receptors in the thalamus. Journal of Pharmacology and Experimental Therapeutics. 2008; 326:475-482. [PubMed: 18477766]

Jia X, Yan J, Xia J, Xiong J, Wang T, Chen Y, et al. Arousal effects of orexin A on acute alcohol intoxication-induced coma in rats. Neuropharmacology. 2012; 62:775-783. [PubMed: 21924278]

Jochum T, Boettger MK, Burkhardt C, Juckel G, Bar KJ. Increased pain sensitivity in alcohol withdrawal syndrome. European Journal of Pain. 2010; 14:713-718. [PubMed: 20018536]

Kash TL. The role of biogenic amine signaling in the bed nucleus of the stria terminals in alcohol abuse. Alcohol. 2012; 46:303-308. [PubMed: 22449787]

Kliethermes CL. Anxiety-like behaviors following chronic ethanol exposure. Neuroscience \& Biobehavioral Reviews. 2005; 28:837-850. [PubMed: 15642625]

Klink R, de Kerchove, d'Exaerde A, Zoli M, Changeux JP. Molecular and physiological diversity of nicotinic acetylcholine receptors in the midbrain dopaminergic nuclei. Journal of Neuroscience. 2001; 21:1452-1463. [PubMed: 11222635]

Koob GF. Drugs of abuse: anatomy, pharmacology and function of reward pathways. Trends in Pharmacological Sciences. 1992; 13:177-184. [PubMed: 1604710]

Kornetsky C, Bain GT, Unterwald EM, Lewis MJ. Brain stimulation reward: effects of ethanol. Alcoholism: Clinical and Experimental Research. 1988; 12:609-616.

Korotkova TM, Ponomarenko AA, Brown RE, Haas HL. Functional diversity of ventral midbrain dopamine and GABAergic neurons. Molecular Neurobiology. 2004; 29:243-259. [PubMed: 15181237]

Lammel S, Hetzel A, Hackel O, Jones I, Liss B, Roeper J. Unique properties of mesoprefrontal neurons within a dual mesocorticolimbic dopamine system. Neuron. 2008; 57:760-773. [PubMed: 18341995]

Lindholm S, Ploj K, Franck J, Nylander I. Repeated ethanol administration induces short- and longterm changes in enkephalin and dynorphin tissue concentrations in rat brain. Alcohol. 2000; 22:165-171. [PubMed: 11163124]

Lu J, Jhou TC, Saper CB. Identification of wake-active dopaminergic neurons in the ventral periaqueductal gray matter. Journal of Neuroscience. 2006; 26:193-202. [PubMed: 16399687]

Margolis EB, Fields HL, Hjelmstad GO, Mitchell JM. Delta-opioid receptor expression in the ventral tegmental area protects against elevated alcohol consumption. Journal of Neuroscience. 2008; 28:12672-12681. [PubMed: 19036960]

Margolis EB, Lock H, Hjelmstad GO, Fields HL. The ventral tegmental area revisited: is there an electrophysiological marker for dopaminergic neurons? Journal of Physiology. 2006; 577:907924. [PubMed: 16959856]

Meloni EG, Gerety LP, Knoll AT, Cohen BM, Carlezon WA Jr. Behavioral and anatomical interactions between dopamine and corticotropinreleasing factor in the rat. Journal of Neuroscience. 2006; 26:3855-3863. [PubMed: 16597740]

Mendes-Gomes J, Amaral VC, Nunes-de-Souza RL. Ventrolateral periaqueductal gray lesion attenuates nociception but does not change anxietylike indices or fear-induced antinociception in mice. Behavioural Brain Research. 2011; 219:248-253. [PubMed: 21238499]

Mendes-Gomes J, Nunes-de-Souza RL. Anxiolytic-like effects produced by bilateral lesion of the periaqueductal gray in mice: influence of concurrent nociceptive stimulation. Behavioural Brain Research. 2009; 203:180-187. [PubMed: 19410607]

Morales-Mulia M, Estrada-Camarena E, Amaya MI, Mejia-Mauries S, Sollozo-Dupont I, Mengod G, et al. Anxiolytic effects of ethanol are partially related to a reduced expression of adenylyl cyclase 5 but not to m-opioid receptor activation in rat nucleus accumbens. Behavioural Brain Research. 2012; 235:189-194. [PubMed: 22963992]

Morean ME, Corbin WR. Subjective response to alcohol: a critical review of the literature. Alcoholism: Clinical and Experimental Research. 2010; 34:385-395.

Peris LC, Peiro AM, Hernandez PZ, de la Parte JF. Environmental and intraperitoneal ethanol influences morphine antinociceptive effect in mice. Life Sciences. 2005; 77:627-634. [PubMed: 15921994] 
Roberto M, Schweitzer P, Madamba SG, Stouffer DG, Parsons LH, Siggins GR. Acute and chronic ethanol alter glutamatergic transmission in rat central amygdala: an in vitro and in vivo analysis. Journal of Neuroscience. 2004; 24:1594-1603. [PubMed: 14973247]

Sharko AC, Kaigler KF, Fadel JR, Wilson MA. Individual differences in voluntary ethanol consumption lead to differential activation of the central amygdala in rats: relationship to the anxiolytic and stimulant effects of low dose ethanol. Alcoholism: Clinical and Experimental Research. 2013; 37(Suppl. 1):E172-E180.

Silberman Y, Matthews RT, Winder DG. A corticotropin releasing factor pathway for ethanol regulation of the ventral tegmental area in the bed nucleus of the stria terminalis. Journal of Neuroscience. 2013; 33:950-960. [PubMed: 23325234]

Spiacci A, Coimbra NC, Zangrossi H. Differential involvement of dorsal raphe subnuclei in the regulation of anxiety- and panic-related defensive behaviors. Neuroscience. 2012; 227:350-360. [PubMed: 23041762]

Theile JW, Morikawa H, Gonzales RA, Morrisett RA. Ethanol enhances GABAergic transmission onto dopamine neurons in the ventral tegmental area of the rat. Alcoholism: Clinical and Experimental Research. 2008; 32:1040-1048.

Theile JW, Morikawa H, Gonzales RA, Morrisett RA. GABAergic transmission modulates ethanol excitation of ventral tegmental area dopamine neurons. Neuroscience. 2011; 172:94-103. [PubMed: 20974231]

Walker BM, Koob GF. Pharmacological evidence for a motivational role of kappa-opioid systems in ethanol dependence. Neuropsychopharmacology. 2008; 33:643-652. [PubMed: 17473837]

Walker BM, Zorrilla EP, Koob GF. Systemic k-opioid receptor antagonism by nor-binaltorphimine reduces dependence-induced excessive alcohol self-administration in rats. Addiction Biology. 2011; 16:116-119. [PubMed: 20579007]

Wills TA, Klug JR, Silberman Y, Baucum AJ, Weitlauf C, Colbran RJ, et al. GluN2B subunit deletion reveals key role in acute and chronic ethanol sensitivity of glutamate synapses in bed nucleus of the stria terminalis. Proceedings of the National Academy of Sciences of the United States of America. 2012; 109:E278-E287. [PubMed: 22219357]

Wise RA. Role of brain dopamine in food reward and reinforcement. Philosophical Transactions of the Royal Society B: Biological Sciences. 2006; 361:1149-1158.

Yang J, Li P, Liang JY, Pan YJ, Yan XQ, Yan FL, et al. Oxytocin in the periaqueductal grey regulates nociception in the rat. Regulatory Peptides. 2011; 169:39-42. [PubMed: 21545817] 


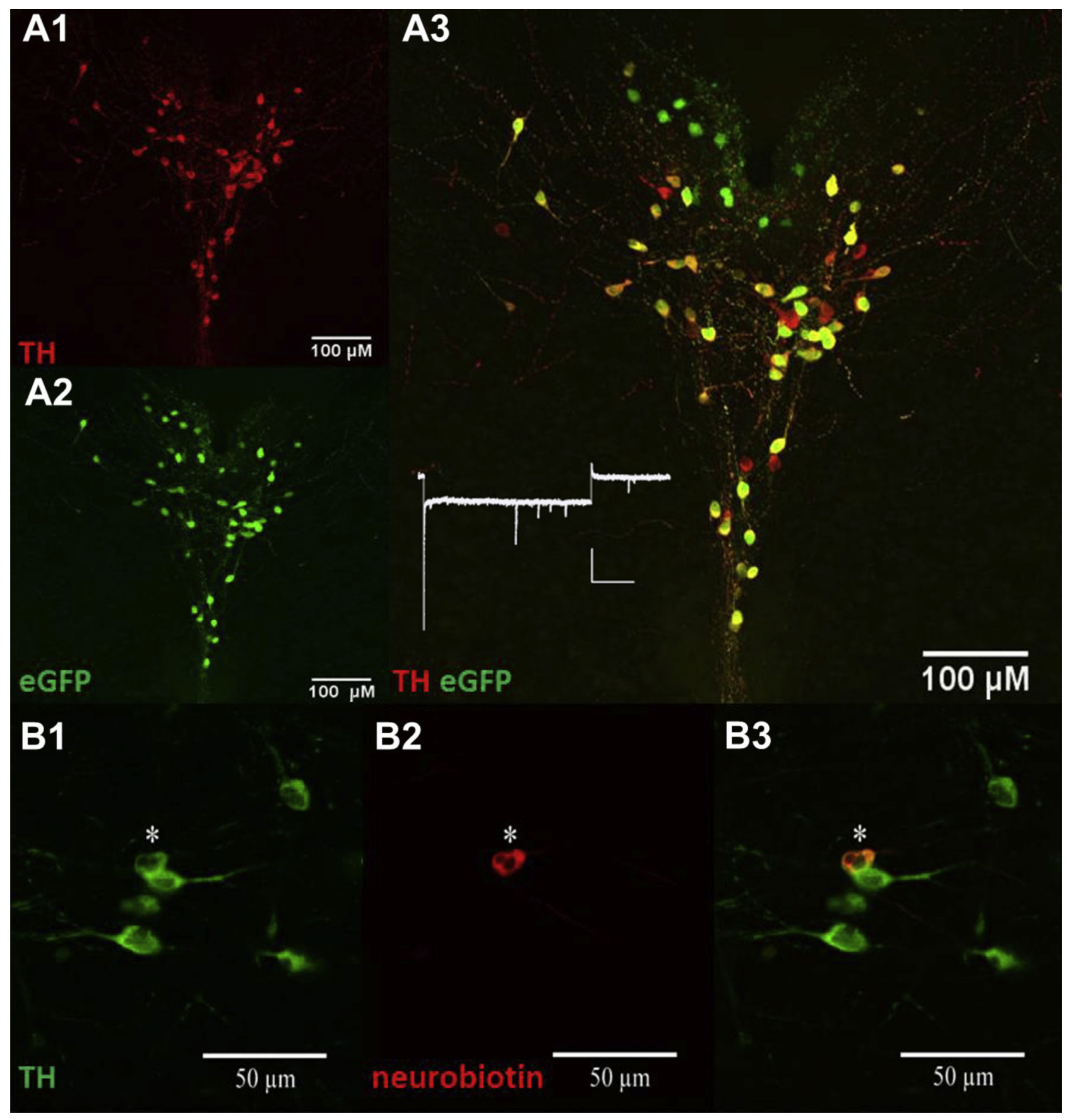

Fig. 1.

The tyrosine hydroxylase-eGFP transgenic mouse model identifies dopamine neurons via fluorescence in the vPAG. (A) Immunohistochemistry can be used to label and verify validity of the reporter via (1) tyrosine hydroxylase fluorescence, (2) eGFP fluorescence, and (3) the co-localization of eGFP tyrosine hydroxylase in dual-positive cells. No significant $I_{h}$ current was observed in vPAG-DA neurons. (B) (1) eGFP and tyrosine hydroxylase-positive cells can be visualized under a microscope to selectively record from. Staining showed that the recorded cells loaded with (2) neurobiotin via diffusion from the patching pipette, are (3) tyrosine hydroxylase positive. 
A i Control

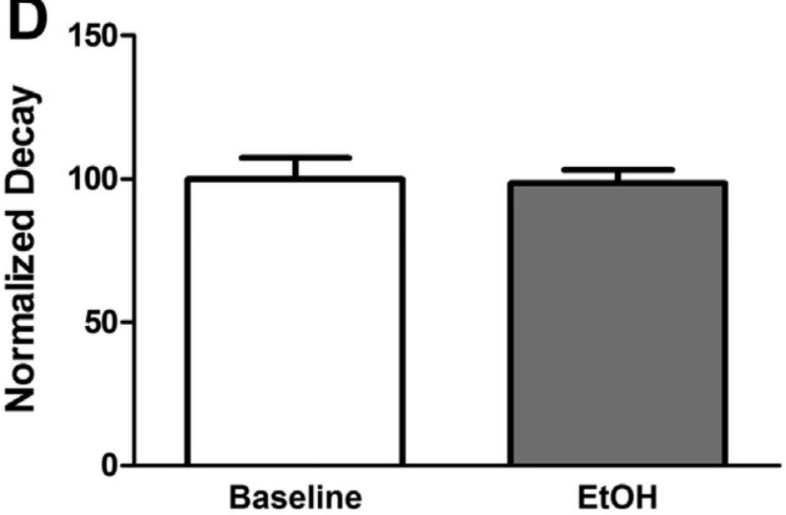

Fig. 2. ii EtOH

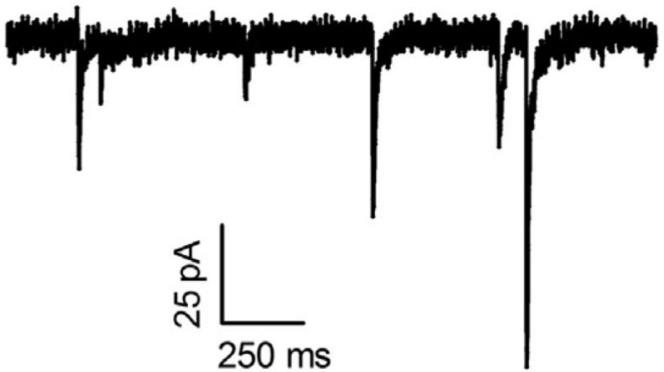

C

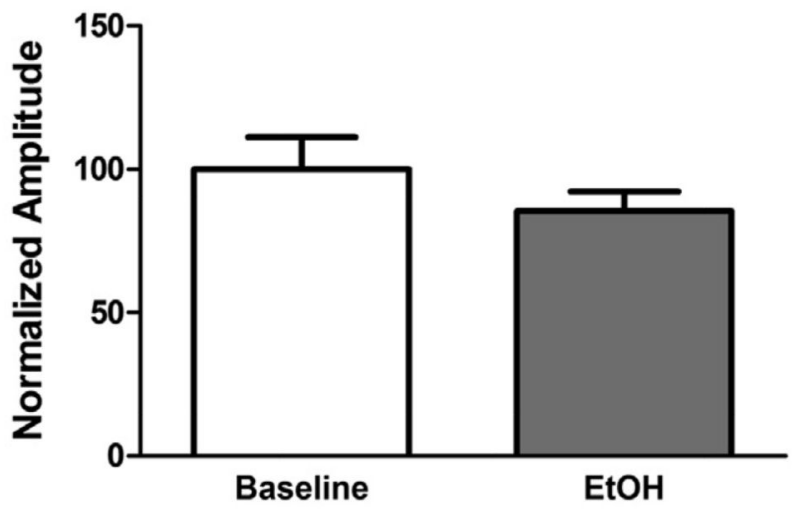

Noise Shift

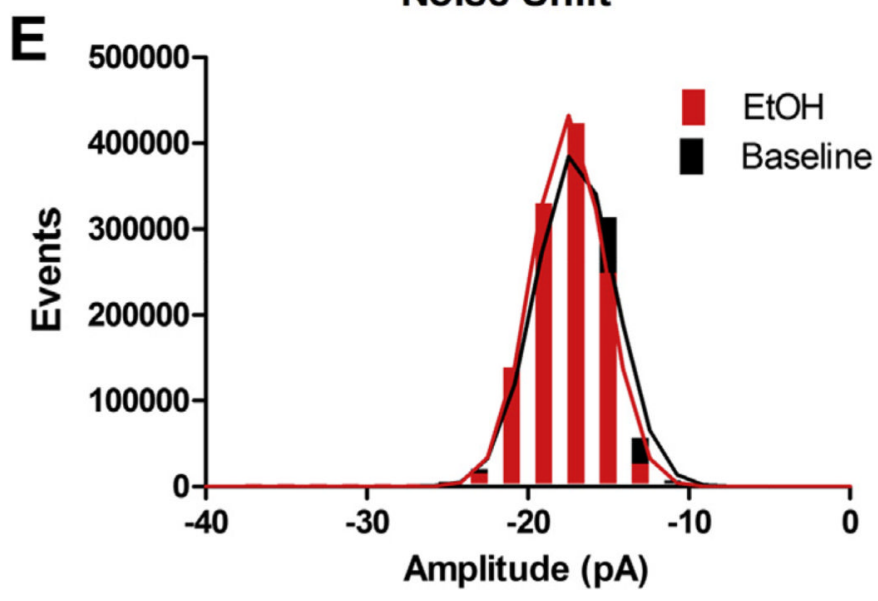

Acute bath-applied alcohol had no effects on mini-inhibitory post-synaptic currents (mIPSCs) in vPAG-dopamine neurons. (A) Representative mIPSC traces of baseline control (i) and after 10 min of alcohol wash-on (ii). (B) Acute alcohol had no effects on mIPSC frequency. (C) Acute alcohol had no effects on mIPSC amplitude. (D) Acute alcohol had no effects on mIPSC decay. (E) Acute alcohol had no effects on mIPSC noise. 
A

C

ปे
Control

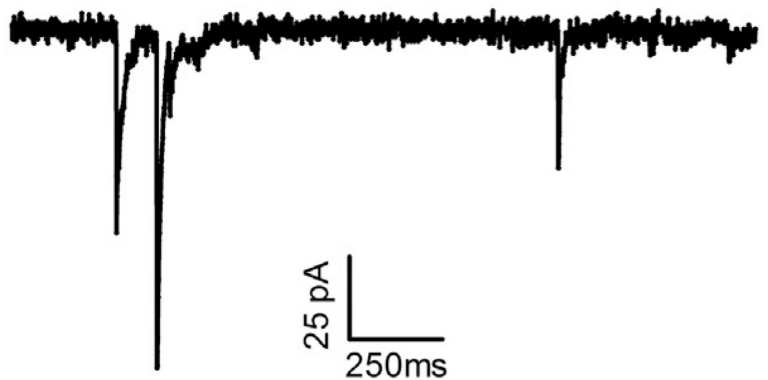

2007
B

EtOH
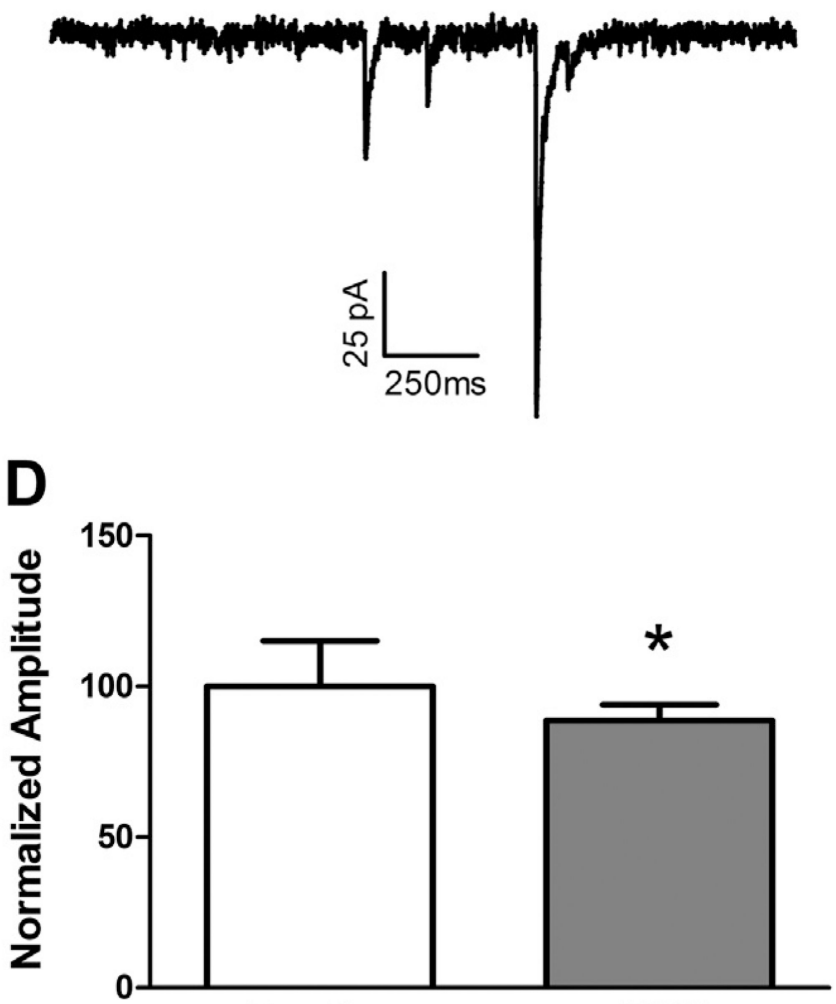

Baseline

Fig. 3.

Acute bath-applied alcohol had no effects on spontaneous inhibitory post-synaptic current (sIPSCs) frequency in vPAG-dopamine neurons. Representative sIPSC traces of (A) baseline control and (B) after 10 min of alcohol wash-on. (C) Acute alcohol had no effects on sIPSC frequency. (D) Acute alcohol significantly decreased sIPSC amplitude. * denotes statistical significance of $p$ value $<0.05$. 
A

Control
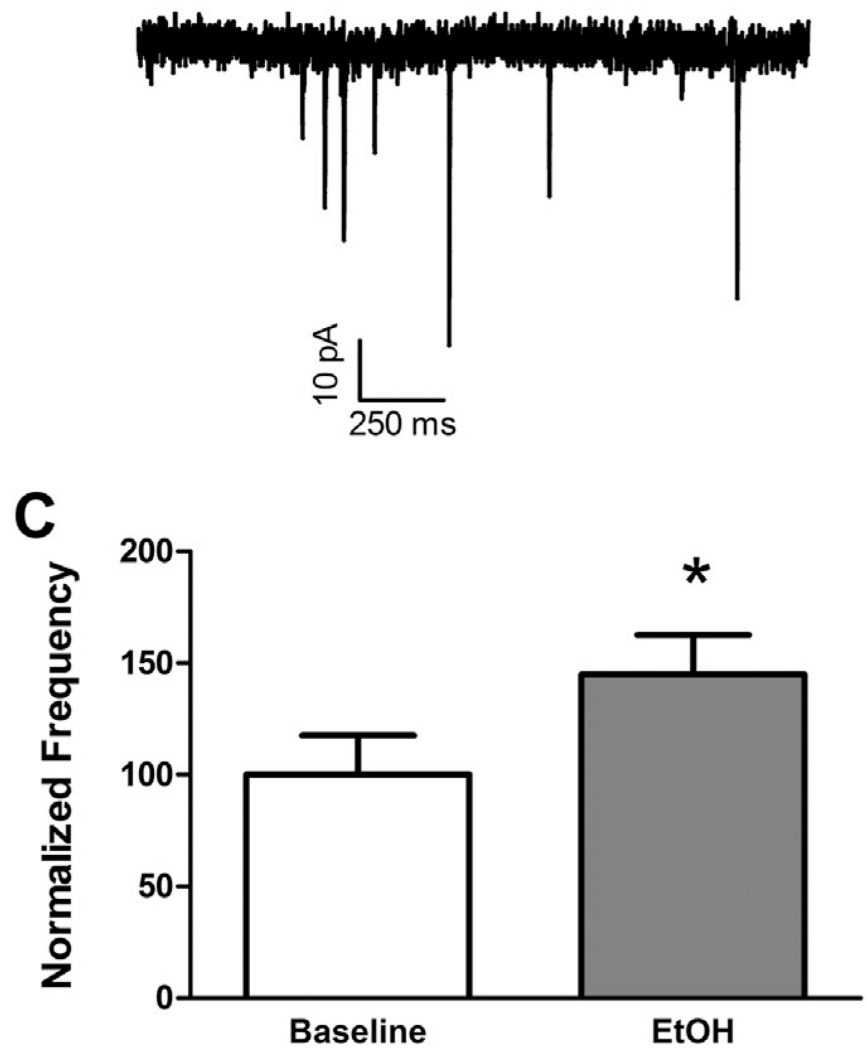

B

EtOH

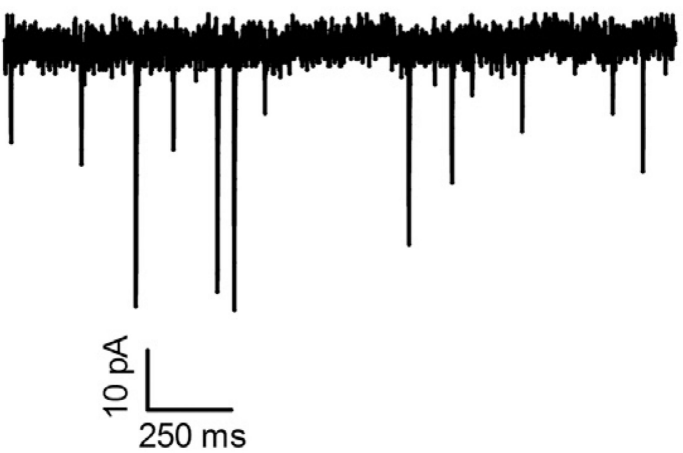

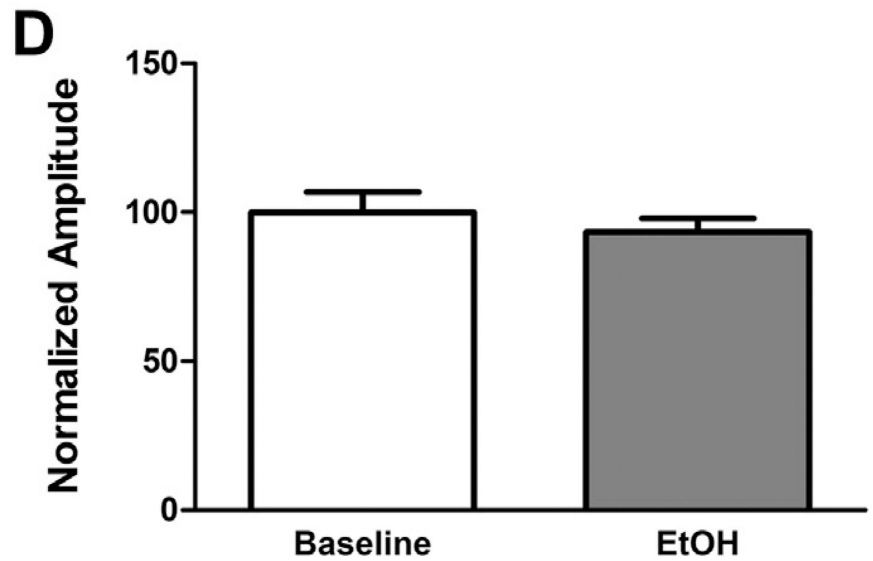

Fig. 4.

Acute bath-applied alcohol increased mini-excitatory post-synaptic currents (mEPSCs) in vPAG-dopamine neurons. (A) Representative mEPSC trace of baseline control. (B)

Representative mEPSC trace after $10 \mathrm{~min}$ of alcohol wash-on. (C) Acute alcohol increased mEPSC frequency. (D) Acute alcohol had no effects on mEPSC amplitude. * denotes statistical significance of $p$ value $<0.05$. 


\section{A Control Firing Rate}

Fig. 5. $p$ value $<0.05$.
B

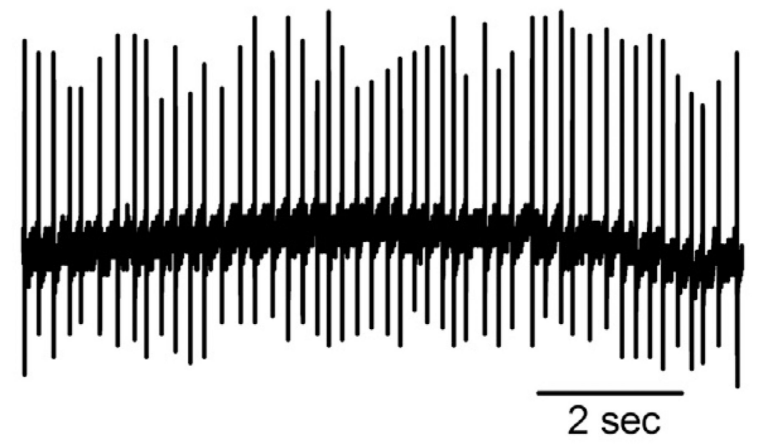

D

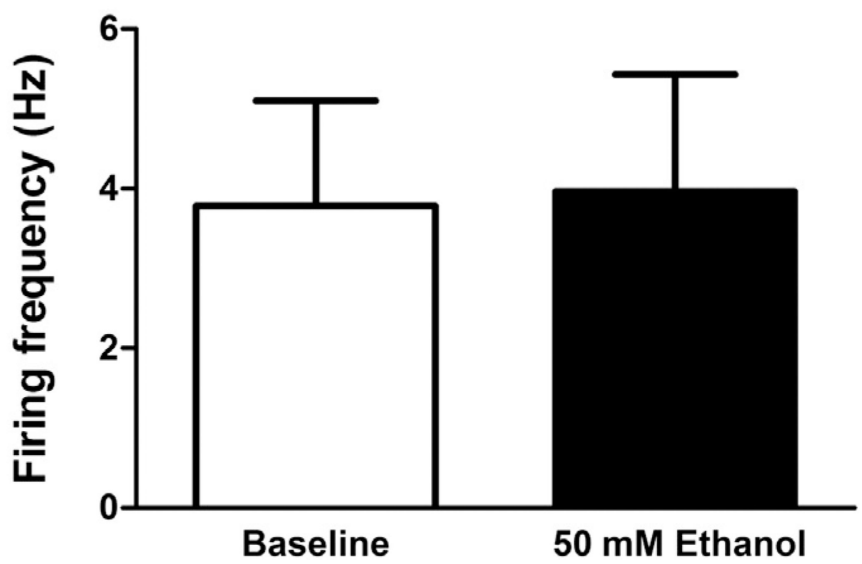

Acute bath-applied alcohol $(50 \mathrm{mM})$ increased firing rate of vPAG-dopamine neurons in a cell-attached recording configuration. (A) Representative firing rate trace of baseline control. (B) Representative firing rate trace in the same neuron after $10 \mathrm{~min}$ of alcohol washon. (C) Alcohol increased firing rate in vPAG-DA neurons. (D) Alcohol had no significant effects on firing rate in the presence of NBQX $(10 \mu \mathrm{M})$. * denotes statistical significance of 
A

control mEPSC

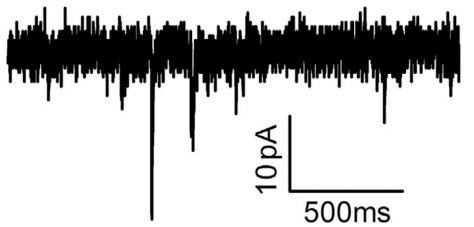

B

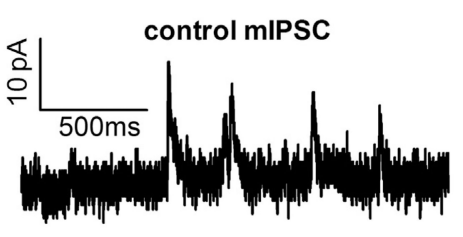

C

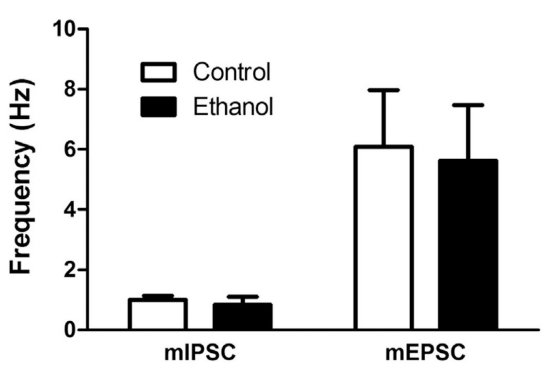

D

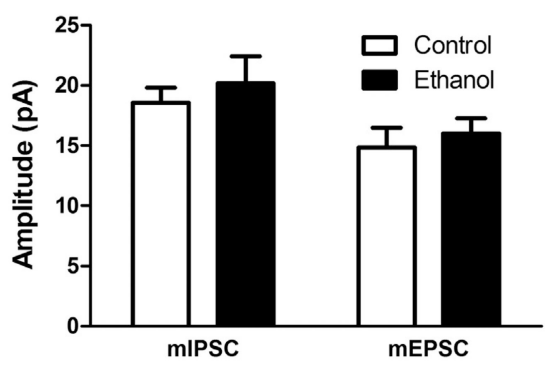

E

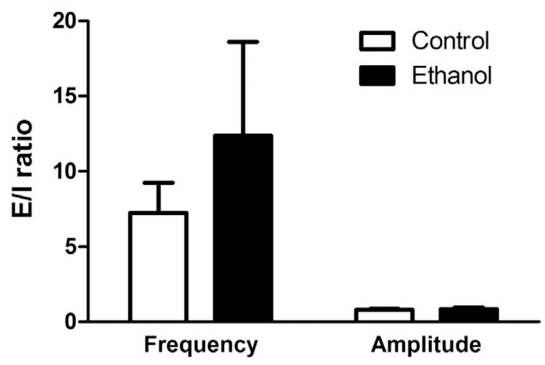

Fig. 6.

Chronic intermittent alcohol vapor exposure did not affect inhibitory and excitatory synaptic properties. (A) Representative mEPSC trace. (B) Representative mIPSC trace. (C) Chronic intermittent alcohol had no effects on mini-frequency. (D) Chronic intermittent alcohol had no effects on mini-amplitude. (E) Chronic intermittent alcohol had no effects on miniexcitatory/mini-inhibitory transmission ratio. 